Semi-theoretical approach for energy dissipation estimation at hydraulic jumps in rough sloped channels

MICHELE PALERMO (IAHR Member), Researcher, DESTEC - Department of Energy,

Systems, Territory and Construction Engineering, University of Pisa, Pisa, Italy

Email: michele.palermo@ing.unipi.it (author for correspondence)

STEFANO PAGLIARA (IAHR Member), Professor, DESTEC - Department of Energy, Systems, Territory and Construction Engineering, University of Pisa, Pisa, Italy

Email:s.pagliara@ing.unipi.it

Running Head: Semi-theoretical approach for energy dissipation estimation 


\title{
Semi-theoretical approach for energy dissipation estimation at hydraulic jumps in rough sloped channels
}

\begin{abstract}
Hydraulic jumps cause a significant flow energy dissipation and generally occur in correspondence with hydraulic structures. Nevertheless, the dissipative mechanisms occurring in correspondence with this hydraulic phenomenon still requires investigations. There are no systematic studies analysing the hydraulic jump energy dissipation for a large range of hydraulic and geometric conditions. To the authors' knowledge, no studies are present in the literature furnishing a complete and exhaustive analysis of the dissipative mechanism for rough sloped channels. This study proposes a novel semitheoretical and general approach to estimate energy dissipation at hydraulic jumps in rough sloped rectangular channels, involving a large range of both hydraulic conditions and bed configurations. Two relationships were derived and their predictive capability has been tested with approximately 500 experimental data from different studies.
\end{abstract}

Keywords: Bed roughness; Energy dissipation; Hydraulic jumps; Hydraulic models; Hydraulic resistance.

\section{Introduction}

Hydraulic jump is one of the main hydraulic phenomena and it can occur in correspondence with many hydraulic structures, including low-head structures, such as block ramps, grade control structures, rock sills and rock weirs (among others, Bhuiyan, Habibzadeh, Rajaratnam, \& Zhu, 2011; Bormann \& Julien, 1991; Farhoudi \& Smith, 1985; Guan, Melville, \& Friedrich, 2014; Pagliara, Mahmoudi Kurdistani, Palermo, \& Simoni, 2016; Pagliara, Palermo \& Das, 2016; Pagliara, Sagvand Hassanabadi, \& Mahmoudi Kurdistani, 2015; Scurlock, Thornton, \& Abt, 2012).

Nevertheless, the effects of bed roughness and channel slope on the dissipative process are still less explored topics. Many studies analysed hydraulic jump characteristics on horizontal rough beds (among others, Carollo, Ferro, \& Pampalone, 2007; Ead \& Rajaratnam, 2002; Felder \& Chanson, 2016; Hughes \& Flack, 1984; Leutheusser \& Schiller, 1975; and Pagliara, Lotti, \& Palermo, 2008), but none of them furnished a general and comprehensive analysis of energy dissipation in a large range of hydraulic conditions and bed configurations. The mentioned studies mainly focused on the effect of different flow bed resistances induced by rough beds on sequent depth ratio. Only very recently, Pagliara \& Palermo (2015) and Felder \& Chanson (2016) have conducted studies to highlight air entrainment contribution on the effective water depths. 
Further studies were also conducted to analyse hydraulic jump characteristics on smooth adverse-sloped beds (McCorquodale \& Mohamed, 1994; Pagliara \& Peruginelli, 2000; Okada \& Aki, 1955; Stevens, 1942). In particular, Pagliara \& Palermo (2015) extended the findings of earlier studies to rough beds (Fig. 1c), concluding that the conjugate depth ratio $Y=y_{2} / y_{1}$ reduces with both relative roughness $k_{s} / k$ and channel bed slope $i=\tan \alpha$, where $k_{s}$ is the roughness height (assumed equal to the mean diameter of the channel bed material $\left.d_{50}\right), k$ is the critical depth, and $\alpha$ is the channel bed inclination with respect to the horizontal (positive for sloping channels and negative for adverse-sloped channels).

Similarly, B- and D-jumps on smooth sloping channels (Fig. 1a) were deeply analysed by several authors (among others, Bakhmeteff \& Matzke, 1938; Chow, 1959; Kawagoshi \& Hager, 1990; Ohtsu \& Yasuda, 1991; Rajaratnam, 1966, 1967). Nevertheless, to the authors' knowledge there are only two studies dealing with hydraulic jump properties on sloping rough beds. In particular, Carollo, Ferro, \& Pampalone (2013) analysed the Bjump, whereas Palermo \& Pagliara (2017) extended the semi-theoretical approach proposed by Pagliara \& Palermo (2015) to D-jumps.

Figure 1 shows all the mentioned configurations, along with the main geometric parameters, i.e., the effective conjugate depths $y_{1}$ and $y_{2}$, the jump length $L_{j}$, the effective top (ET) location (virtual channel bed level), and the vertical distances $z_{1}$ and $z_{2}$ from a reference horizontal plane $(z=0 \mathrm{~m})$ to either the channel bed or ET level at sections 1 and 2, respectively. The horizontal bed configuration is a particular case of those illustrated in Fig. 1, as it occurs for $i=0$. Figure 2 shows two pictures illustrating hydraulic jumps on rough sloping channel (Fig. 2a) and on rough adverse-sloped channel (Fig. 2b), respectively.

But there are no studies dealing with energy dissipation occurring on sloped rough beds. Therefore, the aim of this paper is to analyse the dissipative process in a large range of both boundary conditions and channel configurations. Namely, approximately 500 experimental data were carefully analysed. A novel semi-theoretical approach is proposed to compute the relative energy dissipation occurring at hydraulic jumps, involving both bed roughness and channel slope effects. The findings of Pagliara \& Palermo (2015) and Palermo \& Pagliara (2017) were used to estimate the conjugate depth ratio $Y$, as they probably furnish the most general relationships for $Y$ evaluation in the case of rough sloped rectangular beds. Nevertheless, the validity of the proposed approach is general and does not depend on the methodology adopted to estimate the conjugate depth ratio $Y$. To date, this study probably provides the most general relationships for energy dissipation estimation in such a large range of both hydraulic and geometric parameters. 

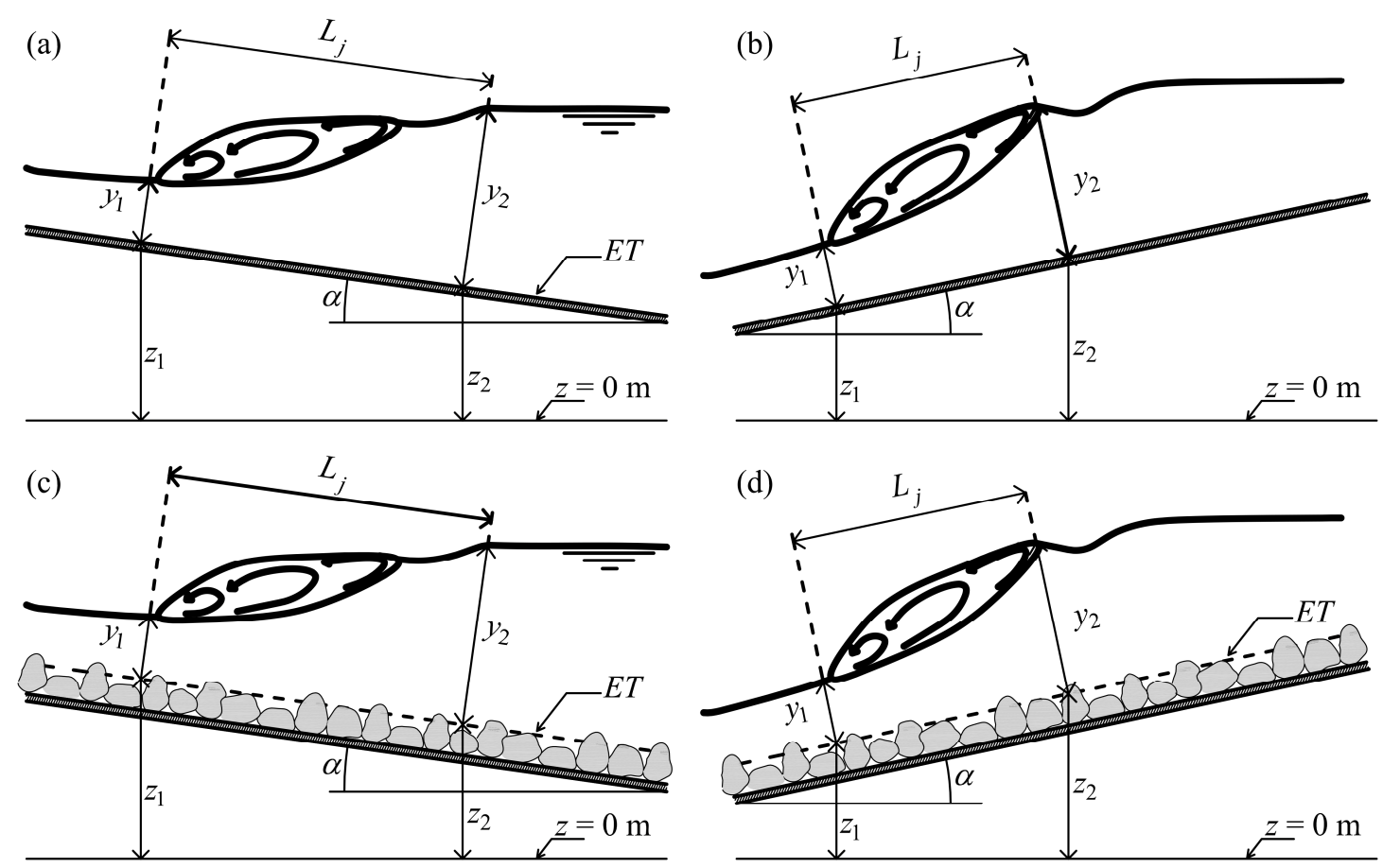

Figure 1 Diagram sketch of a hydraulic jump on (a) smooth sloping bed, (b) smooth adversesloped bed, (c) rough sloping bed and (d) rough adverse-sloped bed.

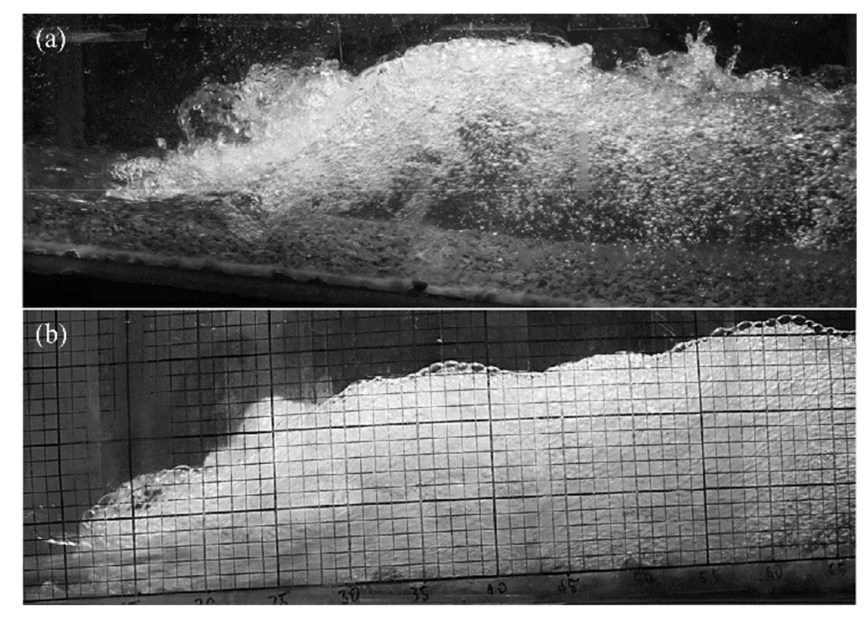

Figure 2 Pictures showing a hydraulic jump on rough (a) sloping and (b) adverse-sloped beds.

\section{Semi-theoretical approach}

For all the geometric configurations and roughness conditions illustrated in Fig. 1, the energy dissipation $E_{1}-E_{2}$ can be computed as follows:

$$
E_{1}-E_{2}=z_{1}+\frac{q^{2}}{2 g y_{1}^{2}}+y_{1} \cos (\alpha)-z_{2}-\frac{q^{2}}{2 g y_{2}^{2}}-y_{2} \cos (\alpha)
$$

where $E_{1}$ and $E_{2}$ are the total energy heads at sections 1 and 2, respectively, and $q$ is the unit discharge. As specified in the previous section, $\alpha$ is positive for sloping channels (Figs 1a and 
1c) and negative for adverse-sloped channels (Figs $1 \mathrm{~b}$ and 1d). In addition, in the case of rough beds, $z_{1}$ and $z_{2}$ are the vertical distances from a reference horizontal plane $(z=0 \mathrm{~m})$ to the ET level at sections 1 and 2, respectively. Based on these assumptions and considering that $L_{j} \sin (\alpha)=z_{1}-z_{2}$, Eq. (1) can be re-arranged as follows:

$$
E_{1}-E_{2}=L_{j} \sin (\alpha)+\frac{q^{2}}{2 g}\left(\frac{1}{y_{1}^{2}}-\frac{1}{y_{2}^{2}}\right)+\left(y_{1}-y_{2}\right) \cos (\alpha)
$$

Note that according to McCorquodale \& Mohamed (1994), Okada \& Aki (1955), Pagliara \& Peruginelli (2000) and Pagliara \& Palermo (2015), hydraulic jump length $L_{j}$ can be assumed coincident with the roller length $L_{r}$ for hydraulic jumps on adverse-sloped beds, i.e., $L_{j} \approx L_{r}$. By dividing both the members of Eq. (2) by $y_{1} \cos (\alpha)$, we obtain:

$$
\frac{E_{1}-E_{2}}{y_{1} \cos (\alpha)}=\frac{L_{j} \sin (\alpha)}{y_{1} \cos (\alpha)}+\frac{q^{2}}{2 g y_{1} \cos (\alpha)}\left(\frac{1}{y_{1}^{2}}-\frac{1}{y_{2}^{2}}\right)+\left(1-\frac{y_{2}}{y_{1}}\right)
$$

Nevertheless, considering that:

$$
E_{1}-z_{1}=\frac{q^{2}}{2 g y_{1}^{2}}+y_{1} \cos (\alpha)
$$

the following equation can be easily derived:

$$
\frac{E_{1}-z_{1}}{y_{1} \cos (\alpha)}=\frac{q^{2}}{2 g y_{1}^{3} \cos (\alpha)}+1
$$

Therefore, by dividing the terms of Eq. (3) by the corresponding terms of Eq. (5), the following Eq. (6) can be obtained:

$$
\frac{\left(E_{1}-E_{2}\right) y_{1} \cos (\alpha)}{\left(E_{1}-z_{1}\right) y_{1} \cos (\alpha)}=\frac{\frac{L_{j} \sin (\alpha)}{y_{1} \cos (\alpha)}+\frac{q^{2}}{2 g y_{1}^{3} \cos (\alpha)}\left(1-\frac{y_{1}^{2}}{y_{2}^{2}}\right)+\left(1-\frac{y_{2}}{y_{1}}\right)}{\frac{q^{2}}{2 g y_{1}^{3} \cos (\alpha)}+1}
$$

which can be re-written as follows:

$$
\frac{\left(E_{1}-E_{2}\right)}{\left(E_{1}-z_{1}\right)}=\frac{\frac{L_{j} \sin (\alpha)}{y_{1} \cos (\alpha)}+\frac{q^{2}}{2 g y_{1}^{3} \cos (\alpha)}\left(1-\frac{y_{1}^{2}}{y_{2}^{2}}\right)+\left(1-\frac{y_{2}}{y_{1}}\right)}{\frac{q^{2}}{2 g y_{1}^{3} \cos (\alpha)}+1}
$$

or, equivalently,

$$
\Delta E_{r}=\frac{\left(E_{1}-E_{2}\right)}{\left(E_{1}-z_{1}\right)}=\frac{2 \lambda \tan (\alpha)+\frac{\mathrm{F}_{1}^{2}}{\cos (\alpha)}\left(1-Y^{-2}\right)+2(1-Y)}{\frac{\mathrm{F}_{1}^{2}}{\cos (\alpha)}+2}
$$

where $\Delta E_{r}=\left(E_{1}-E_{2}\right) /\left(E_{1}-z_{1}\right)$ is the relative energy dissipation, $\lambda=L_{j} / y_{1}$ is the relative hydraulic jump length and $F_{1}$ is the appraoching Froude number at section 1. Eq. (8) 
represents the general expression of the relative energy dissipation for all the mentioned bed geometries and boundary conditions.

Pagliara \& Palermo (2015) and Palermo \& Pagliara (2017) extended the thoretical Eq. (9) by Carollo et al. (2007) to both adverse sloped and sloping beds, respectively. Namely, they furnished the following general Eqs (9)-(12) to estimate the conjugate depth ratio $Y$ for $\mathrm{F}_{1}>1.5,-0.3 \leq i \leq 0.15$ and $0 \leq k_{s} / k \leq 0.45$

$$
Y=\frac{-1+\sqrt{1+8 \mathrm{G}_{1}^{2}(1-\beta)}}{2}
$$

where $\mathrm{G}_{1}$ is the jump parameter and $\beta$ is the momentum deficit parameter. According to Pagliara \& Palermo (2015), for $i \leq 0$ (adverse-sloped and horizontal beds), $\mathrm{G}_{1}$ can be computed using the following Eq. (10):

$$
\mathrm{G}_{1}=3.32^{1.52 i} \mathrm{~F}_{1}
$$

Whereas, according to Palermo \& Pagliara (2017), for $i \geq 0$ (sloping and horizontal channels), $\mathrm{G}_{1}$ can be expressed as:

$$
\mathrm{G}_{1}=3.32^{2.7 i} \mathrm{~F}_{1}
$$

Furthermore, Palermo \& Pagliara (2017) showed that the momentum deficit parameter $\beta$ can be computed with Eq. (12) for both adverse-sloped and sloping rough beds, as it only depends on the relative roughness:

$$
\beta=-0.14\left[1-\exp \left(2.38 \frac{k_{s}}{k}\right)\right]
$$

where $k_{s}$ is the roughness heigth, which can be assumed equal to the mean diameter of the bed material $d_{50}$ in the case of uniform gravel bed (Hughes \& Flack, 1984; Carollo et al., 2007). Finally, Pagliara \& Palermo (2015) and Palermo \& Pagliara (2017) showed that the relative hyraulic jump length $L_{j}$ can be computed with Eq. (13), where $Y$ is evaluated with Eq. (9):

$$
\lambda=4.616(Y-1)
$$

Therefore, by substituting the expressions of $Y$ and $\lambda$ given by Eqs (9) and (13) in Eq. (8), $\Delta E_{r}$ can be easily estimated for all the mentioned configurations and channel bed boundary conditions. Nevertheless, especially for sloping channels, it could be more convenient to compute the relative energy dissipation $\Delta E_{r}{ }^{*}=\left(E_{1}-E_{2}\right) /\left(E_{1}-z_{2}\right)$. Therefore, with similar analytical steps, the following expression can be derived:

$$
\Delta E_{r}^{*}=\frac{\left(E_{1}-E_{2}\right)}{\left(E_{1}-z_{2}\right)}=\frac{2 \lambda \tan (\alpha)+2(1-Y)+\frac{\mathrm{F}_{1}^{2}}{\cos (\alpha)}\left(1-Y^{-2}\right)}{\frac{\mathrm{F}_{1}^{2}}{\cos (\alpha)}+2+2 \lambda \tan (\alpha)}
$$


where $Y$ and $\lambda$ can be computed with Eq. (9) and (13), respectively. Equation (14) is an alternative general expression for the relative energy dissipation and is valid for $F_{1}>1.5,-0.3$ $\leq i \leq 0.15$ and $0 \leq k_{s} / k \leq 0.45$. It should be noted that Eq. (8) and Eq. (14) are analytically coincident for $i=0$. In addition, they coincides with the well-known expression valid for smooth horizontal beds when $i=0$ and $k_{s} / k=0$.

\section{Results and discussion}

The proposed semi-theoretical approach was validated by using approximately 500 experimental data derived from different authors. Namely, the preliminary validation of Eq. (8) was conducted by steps. Available data relative to horizontal rough beds were grouped according to their relative roughness values $k_{s} / k$. In particular, data were grouped for $0 \leq k_{s} / k$ $\leq 0.10,0.10<k_{s} / k \leq 0.20,0.20<k_{s} / k \leq 0.30,0.30<k_{s} / k \leq 0.40$, and $0.40<k_{s} / k \leq 0.50$. Then, experimental data were plotted in a graph $\Delta E_{r}$ vs $\mathrm{F}_{1}$ along with Eq. (8). Note that for horizontal beds $i=0$, Eq. (8) was plotted assuming the medium value of each relative roughness group, e.g., $k_{s} / k=0.25$ for $k_{s} / k$ ranging between 0.20 and 0.30 . Figure 3 shows selected examples of comparison between experimental data derived from Ead \& Rajaratnam (2002), Hughes \& Flack (1984) and Pagliara et al. (2008) and Eq. (8). Overall, Fig. 3 shows a good agreement between experimental data and the proposed equation. Furthermore, it is worth noting that the relative energy dissipation increases with relative roughness and approaching Froude number, i.e., $\Delta E_{r}$ is a monotonic increasing function of both $k_{s} / k$ and $\mathrm{F}_{1}$.

This occurrence is mainly due to the fact that shear stresses increase with both $k_{s} / k$ and $\mathrm{F}_{1}$. In fact, the momentum deficit $M_{1}-M_{2}$ (where $M_{1}$ and $M_{2}$ are the momentum fluxes at the sections 1 and 2, respectively) increases with $F_{1}$. But, for otherwise identical conditions, the integrated shear stress $F_{\tau}$ increases with both $k_{s} / k$ and $\mathrm{F}_{1}$. Note that Carollo et al. (2007) stated that $F_{\tau}$ can be expressed as $\beta\left(M_{1}-M_{2}\right)$. Thus, according to Eq. (12), $\beta$ increases with $k_{s} / k$ and $F_{\tau}$ is a monotonic increasing function of both $k_{s} / k$ and $\mathrm{F}_{1}$. This effect is further amplified by the air entrainment. Pagliara \& Palermo (2015) showed that for constant $F_{1}$, the average air concentration increases with $k_{s} / k$, resulting in a more turbulent flow and in an increase of the dissipation rate.

A similar behavior can be also pointed out for both adverse-sloped channels (Fig. 4) and sloping channels (Fig. 5). 

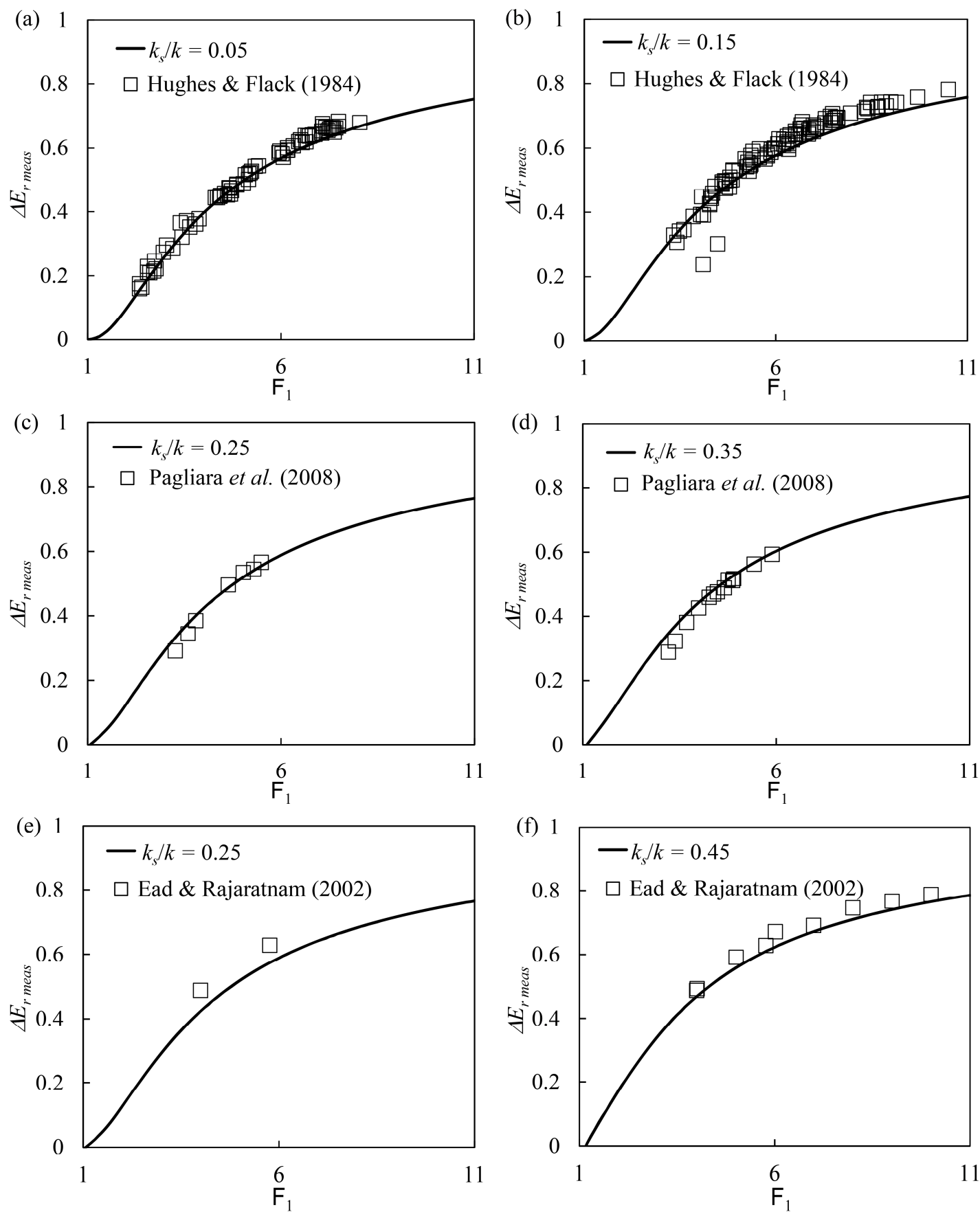

Figure 3 Energy dissipation for horizontal rough beds $(i=0)$ : comparison of experimental data with Eq. (8) for (a) $k_{s} / k=0.05$; (b) $k_{s} / k=0.15$; (c) $k_{s} / k=0.25$; (d) $k_{s} / k=0.35$; (e) $k_{s} / k=$ 0.25 ; and (f) $k_{s} / k=0.45$. 

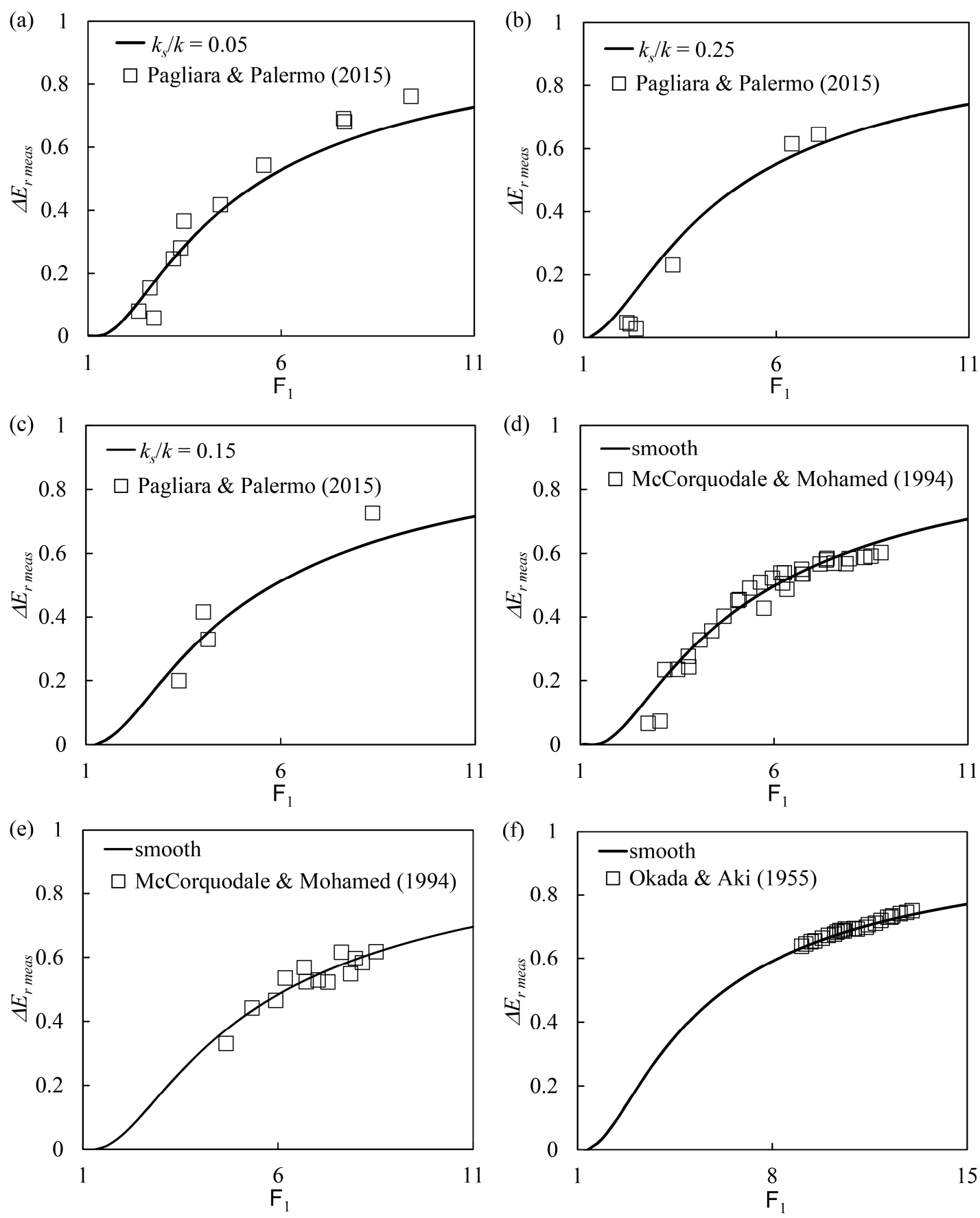

Figure 4 Energy dissipation for adverse-sloped beds $(i<0)$. Comparison of experimental data with Eq. (8) for rough beds: (a) $k_{s} / k=0.05$ and $i=-0.05$; (b) $k_{s} / k=0.25$ and $i=-0.05$; and (c) $k_{s} / k=0.15$ and $i=-0.10$; and for smooth beds: (d) $i=-0.10$; (e) $i=-0.15$; and (f) $i=-0.17$.

Figures 4a-c show comparisons of selected examples of experimental data derived from Pagliara \& Palermo (2015) for adverse-sloped rough beds with Eq. (8), whereas Figs 4d-f show the same for data derived from McCorquodale \& Mohamed (1994) and Okada \& Aki (1955) for adverse-sloped smooth beds. In particular, Figs 4a-b show experimental data for $i$ $=-0.05$ and $k_{s} / k=0.05$ and 0.25 , respectively, along with the plot of Eq. (8), where $Y$ is 
calculated with Eq. (9) and $\mathrm{G}_{1}$ with Eq. (10). Figure 4c shows the same for $k_{s} / k=0.15$ and $i=$ -0.10. Finally, Figs 4d-f compare Eq. (8) with experimental data on smooth adverse-sloped beds for $i=-0.10,-0.15$ and -0.17 , respectively. It can be easily observed that Eq. (8) well represents experimental data trend. In general, a slight deviation can be pointed out for very high relative roughness and low $\mathrm{F}_{1}$. For these conditions, the conjugate depth ratio $Y$ is strongly affected by the parameter $k_{s} / k$ (Carollo et al., 2007; Hughes \& Flack, 1984; and Pagliara \& Palermo, 2015). In particular, Pagliara \& Palermo (2015) showed that $Y$ values are more spread due to eventual rooster tails formation, thus resulting in a slight underestimation of the relative energy dissipation.

Same considerations apply for sloping channels. Figure 5 shows two selected examples of experimental data derived from Palermo \& Pagliara (2017) along with the respective plots of Eq. (8).
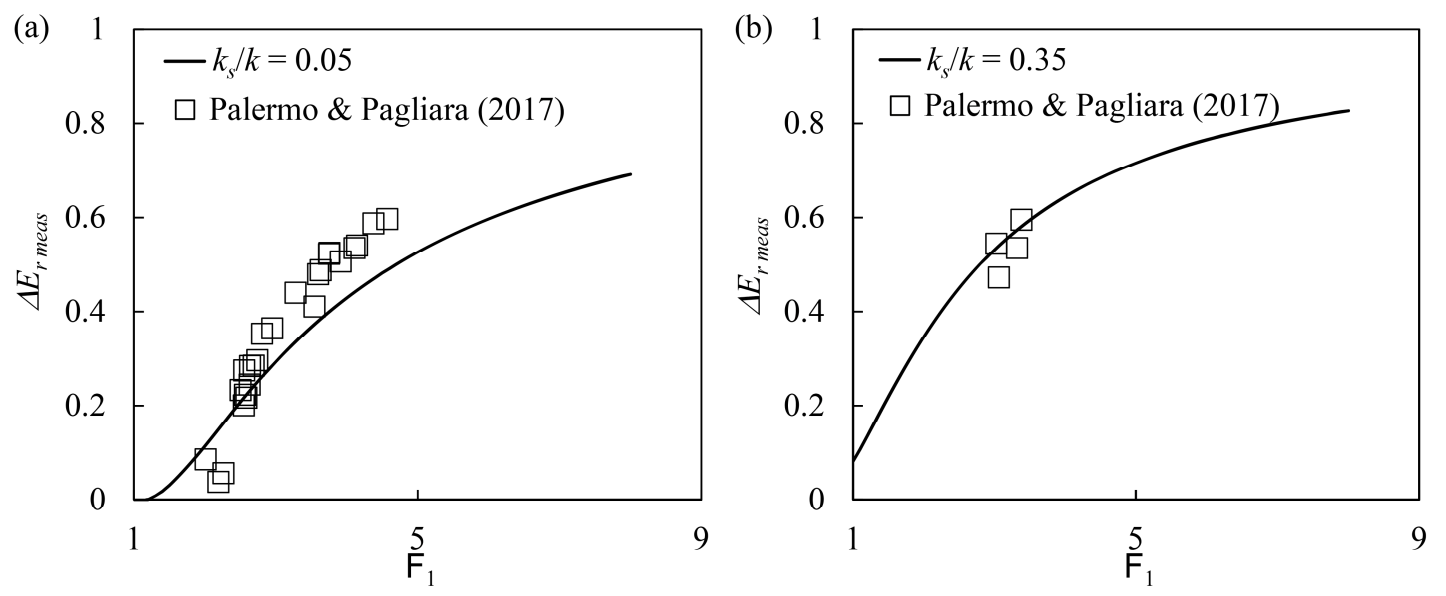

Figure 5 Energy dissipation for sloping rough beds $(i>0)$ : comparison of experimental data with Eq. (8) for: (a) $k_{s} / k=0.05$ and $i=0.05$; and (b) $k_{s} / k=0.35$ and $i=0.15$.

Also in this case, there is a substantial agreement between the semi-theoretical predicting equation and experimental data. Nevertheless, especially for lower $k_{s} / k$, a slight deviation between measured and predicted values of the variable $\Delta E_{r}$ can be pointed out. This slight difference could be due to practical difficulties to correctly assess hydraulic jump length for sloping channels (Kawagoshi \& Hager, 1990; Ohtsu \& Yasuda, 1991). In fact, for adversesloped channels, the hydraulic jump length can be assumed coincident with the roller length, thus resulting in an easily measurable characteristic. Conversely, for sloping channels, hydraulic jump length assessment is more difficult and can cause a slight under- or overestimation of the control volume weight. Nevertheless, for practical applications, this slight deviation can be considered negligible. 
Overall, it can be observed that $\Delta E_{r}$ is a monotonic increasing function of the bed slope $i$ (Fig. 6). This occurrence can be easily explained considering that the conjugate depth ratio $Y$ is a monotonic increasing function of $i$ (Ohtsu \& Yasuda, 1991; Okada \& Aki, 1955; Pagliara \& Palermo, 2015; Palermo \& Pagliara, 2017). In particular, the effect of the variable $i$ on $\Delta E_{r}$ is more significant for sloping channels than for adverse-sloped channels.

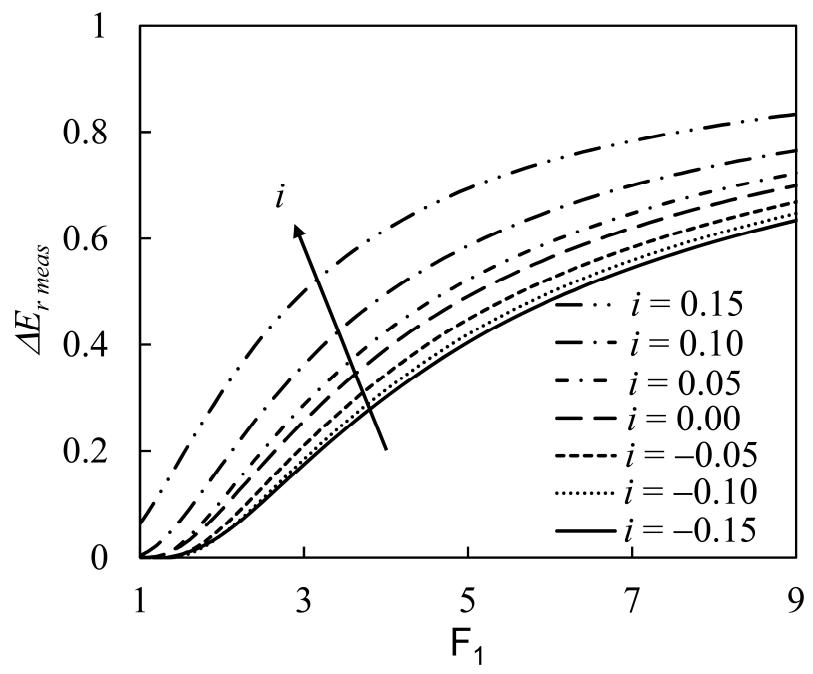

Figure 6 Bed slope effect on the energy dissipation on smooth beds.

Based on the previous observations and preliminary validations, the predictive capability of Eq. (8) was tested using all the available data. For horizontal rough beds (Fig. 7a), both data derived from Hughes \& Flack (1984) and Pagliara et al. (2008) are very well predicted (within 15\% deviation). Conversely, a higher deviation can be pointed out for data derived from Carollo et al. (2007). Nevertheless, also in this last case, most of the data are well estimated by Eq. (8). The higher deviation can be observed for those data characterized by relatively small $y_{1}$ values, for which the measurement uncertainties are more significant. Similarly, Fig. $7 \mathrm{~b}$ shows that Eq. (8) well predicts experimental data derived from McCorquodale \& Mohamed (1994), Okada \& Aki (1955) and Pagliara \& Peruginelli (2000) for smooth adverse-sloped beds. Whereas, a relatively higher deviation can be observed for data relative to both rough adverse-sloped and rough sloping beds (Fig. 7c), particularly for those data characterized by higher $k_{s} / k$ and lower $\mathrm{F}_{1}$ values, for which the eventual formation of rooster tails makes more difficult the assessment of the conjugate depth ratio. Overall, Eq. (8) reasonably well predicts all experimental data and, to the authors' knowledge, is the most general equation present in the literature valid in such a large range of parameters.

Same considerations apply for Eq. (14), whose analytical derivation is essentially identical to that reported for Eq. (8). 

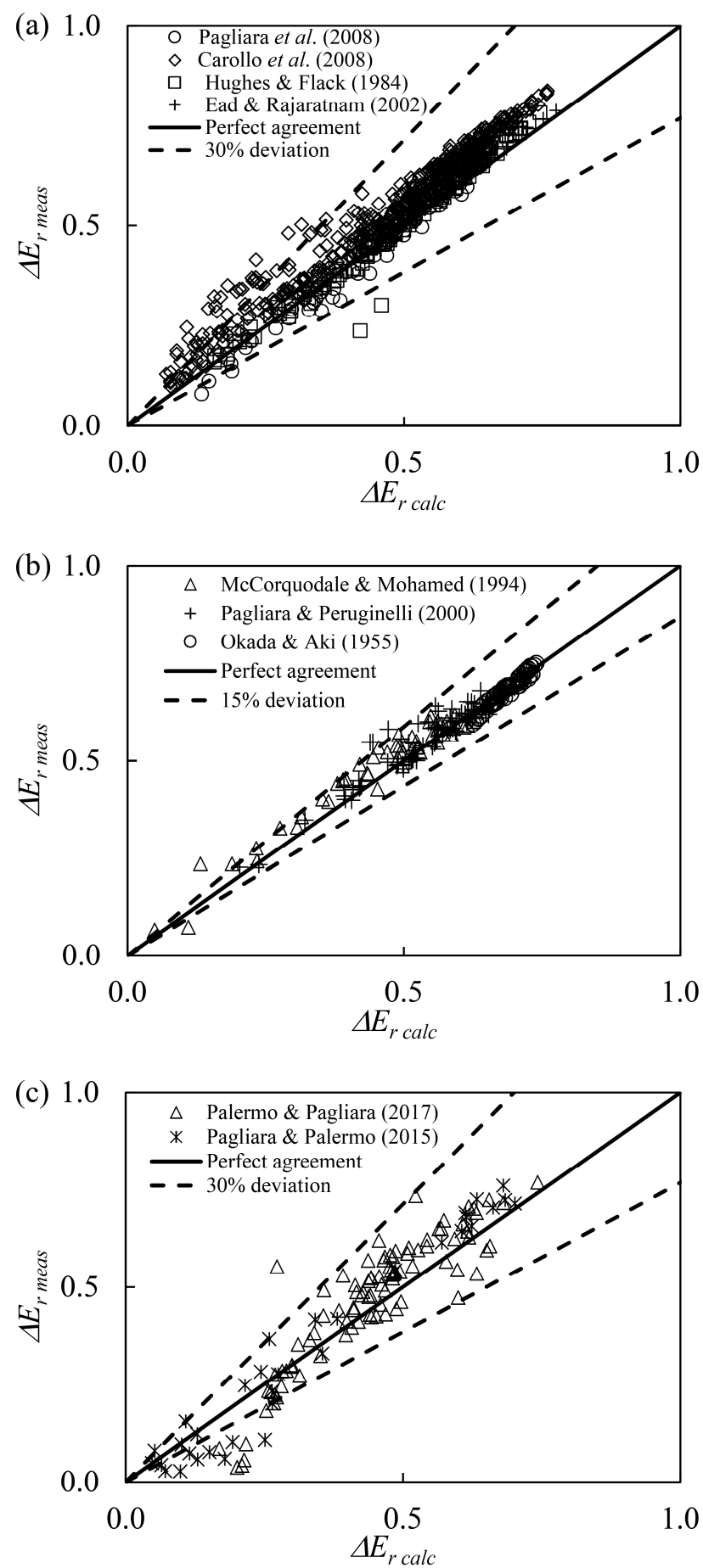

Figure 7 Comparison between measured and computed (with Eq. 8) values of the variable $\Delta E_{r}$ for: (a) horizontal bed $(i=0)$; (b) smooth adverse-sloped bed $(i<0)$; and (c) adverse-sloped and sloping rough beds $(i<0$ and $i>0)$.

\section{Conclusions}

This paper analysed the energy dissipation at hydraulic jumps in rectangular sloped channels. Two general equations were proposed to estimate the relative energy dissipation in a large 
range of hydraulic parameters and geometric conditions. In particular, the proposed general equations are theoretically derived and do not depend on the methodology adopted to estimate the conjugate depth ratio. In the present study, the findings of earlier studies of the same authors were used to estimate $Y$. The predictive capability of theoretical equations was tested by using data from different authors, involving different geometric configurations and boundary conditions, i.e., smooth and rough horizontal, adverse-sloped and sloping rectangular channels. The analysis of experimental data showed that energy dissipation strongly depends on the relative roughness and on the channel bed slope, i.e., it is a monotonic increasing function of both relative roughness and bed slope. To the authors' knowledge, the proposed relationships are the most general present in the literature and can be considered valid tools to estimate the energy dissipation at hydraulic jumps.

\section{Notation}

$d_{50}=$ mean diameter of the bed material $(\mathrm{m})$

$E_{1}=$ total energy head at section $1(\mathrm{~m})$

$E_{2}=$ total energy head at section $2(\mathrm{~m})$

$\mathrm{F}_{1}=$ Froude number at section $1(-)$

$F_{\tau}=$ integrated shear stress per unit width $\left(\mathrm{Nm}^{-1}\right)$

$g=$ gravity acceleration $\left(\mathrm{ms}^{-2}\right)$

$\mathrm{G}_{1}=$ adverse jump parameter (-)

$i=$ channel bed slope (-)

$k=$ critical depth $(\mathrm{m})$

$k_{s}=$ roughness height $(\mathrm{m})$

$L_{j}=$ hydraulic jump length (m)

$L_{r}=$ length of roller (m)

$M_{1}=\rho y_{1} U_{1}^{2}$ momentum flux per unit width at section $1\left(\mathrm{Nm}^{-1}\right)$

$M_{2}=\rho y_{2} U_{2}^{2}$ momentum flux per unit width at section $2\left(\mathrm{Nm}^{-1}\right)$

$q=$ discharge per unit width $\left(\mathrm{m}^{2} \mathrm{~s}^{-1}\right)$

$U_{1}=$ average velocity at section $1\left(\mathrm{~ms}^{-1}\right)$

$U_{2}=$ average velocity at section $2\left(\mathrm{~ms}^{-1}\right)$

$Y=y_{2} / y_{1}$ sequent depth ratio (-)

$y_{1}=$ effective upstream depth of the hydraulic jump (m)

$y_{2}=$ effective downstream depth of the hydraulic jump (m)

$z_{1}=$ vertical distance from a reference horizontal plane to channel bed at section 1 (m) 
$z_{2}=$ vertical distance from a reference horizontal plane to channel bed at section $2(\mathrm{~m})$

$\alpha=$ angle of the bed slope respect to horizontal (rad)

$\beta=$ momentum deficit parameter (-)

$\gamma=$ specific weight $\left(\mathrm{Nm}^{-3}\right)$

$\Delta E_{r}=\left(E_{1}-E_{2}\right) /\left(E_{1}-z_{1}\right)$ relative energy dissipation (-)

$\Delta E_{r}{ }^{*}=\left(E_{1}-E_{2}\right) /\left(E_{1}-z_{2}\right)$ relative energy dissipation (-)

$\rho=$ water density $\left(\mathrm{kgm}^{-3}\right)$

$\lambda=$ non-dimensional length of the jump (-)

\section{References}

Bakhmeteff, B. A., \& Matze, A. E. (1938). The hydraulic jump in sloped channels. Transactions ASCE, 60(HYD-60-1), 111-118.

Bhuiyan, F., Habibzadeh, N., Rajaratnam, N., \& Zhu, D. Z. (2011). Reattached turbulent submerged offset jets on rough beds with shallow tailwater. Journal of Hydraulic Engineering, 137(12).

Bormann, N. E., \& Julien, P. Y. (1991). Scour downstream of grade-control structures. Journal of Hydraulic Engineering, 117(5), 579-594.

Carollo, F. G., Ferro, V., \& Pampalone, V. (2007). Hydraulic jump on rough beds. Journal of Hydraulic Engineering, 133(9), 989-999.

Carollo, F. G., Ferro, V., \& Pampalone, V. (2013). Sequent depth ratio of a B-jump on smooth and rough beds. Journal of Agricultural Engineering, XLIV(e12), 82-86.

Chow, V. T. (1959). Open-channel hydraulics. New York: McGraw-Hill.

Ead, S. A., \& Rajaratnam, N. (2002). Hydraulic jumps on corrugated beds. Journal of Hydraulic Engineering, 128(7), 656-663.

Farhoudi, J., \& Smith, K. V. H. (1985). Local scour profiles downstream of hydraulic jump. Journal of Hydraulic Research, 23(4), 343-358.

Felder, S., \& Chanson, H. (2016). An Experimental Study of Air-water Flows in Hydraulic Jumps with Channel Bed Roughness (Report No. 259). Sydney, Australia: The University of New South Wales.

Guan, D., Melville, B. W., \& Friedrich, H. (2014). Flow patterns and turbulence structures in a scour hole downstream of a submerged weir. Journal of Hydraulic Engineering, 140(1), 68-76.

Kawagoshi, N., \& Hager, W. H. (1990). B-jump in sloping channel II. Journal of Hydraulic Research, 28(4), 461-480.

Hughes, W. C., \& Flack, J. E. (1984). Hydraulic jump properties over a rough bed. Journal of Hydraulic Engineering, 110(12), 1755-1771. 
Leutheusser, H. J., \& Schiller, E. J. (1975). Hydraulic jump in a rough channel. International Water Power \& Dam Construction, 27(5), 186-191.

McCorquodale, J. A., \& Mohamed, M. S. (1994). Hydraulic jumps on adverse slopes. Journal of Hydraulic Research, 32(1), 119-130.

Okada, A., \& Aki, S. (1955). Experimental study of hydraulic jump on reversed slope apron. Journal of Central Research Institute of Electric Power Industry Technical Lab, 5(6).

Ohtsu, I., \& Yasuda, Y. (1991). Hydraulic jump in sloping channels. Journal of Hydraulic Engineering, 117(7), 905-921.

Pagliara, S., Lotti, I., \& Palermo, M. (2008). Hydraulic jumps on rough bed of stream rehabilitation structures. Journal of Hydro-Environment Research, 2(1), 29-38.

Pagliara, S., Mahmoudi Kurdistani, S., Palermo, M., \& Simoni, D. (2016). Scour due to rock sills in straight and curved horizontal channels. Journal of Hydro-Environment Research, 10, 12-20.

Pagliara, S., \& Palermo, M. (2015). Hydraulic jumps on rough and smooth beds: aggregate approach for horizontal and adverse-sloped beds. Journal of Hydraulic Research, 53(2), 243-252.

Pagliara, S., Palermo, M., \& Das, R. (2016). Eco-Friendly Countermeasures for Enlarged Basins Erosion. River Research and Applications, 32(3), 441-451.

Pagliara, S., \& Peruginelli, A. (2000). Limiting and sill-controlled adverse-slope hydraulic jump. Journal of Hydraulic Engineering, 126(11), 847-851.

Pagliara, S., Sagvand Hassanabadi, L., \& Mahmoudi Kurdistani, S. (2015). Clear water scour downstream of $\log$ deflectors in horizontal channels. Journal of Irrigation and Drainage Engineering, 141(9).

Palermo, M., \& Pagliara, S. (2017). D-jump in rough sloping channels at low Froude numbers. Journal of Hydro-Environment Research, 14(1), 150-156.

Rajaratnam, N. (1966). The hydraulic jump in sloping channels. Irrigation and Power, 32(2), 137-149.

Rajaratnam, N. (1967). Hydraulic jumps. Advances in hydroscience (pp. 197-280), San Diego: Academic.

Scurlock, S. M., Thornton, C. I., \& Abt, S. R. (2012). Equilibrium scour downstream of threedimensional grade-control structures. Journal of Hydraulic Engineering, 138(2), 167176.

Stevens, C. J. (1942). Discussion of "The hydraulic jumps in sloping channels". Transactions ASCE, 2228, 1125-1135. 


\section{List of figures}

Figure 1 Diagram sketch of a hydraulic jump on (a) smooth sloping bed, (b) smooth adversesloped bed, (c) rough sloping bed and (d) rough adverse-sloped bed.

Figure 2 Pictures showing a hydraulic jump on rough (a) sloping and (b) adverse-sloped beds.

Figure 3 Energy dissipation for horizontal rough beds $(i=0)$ : comparison of experimental data with Eq. (8) for (a) $k_{s} / k=0.05$; (b) $k_{s} / k=0.15$; (c) $k_{s} / k=0.25$; (d) $k_{s} / k=0.35$; (e) $k_{s} / k=$ 0.25 ; and (f) $k_{s} / k=0.45$.

Figure 4 Energy dissipation for adverse-sloped beds $(i<0)$. Comparison of experimental data with Eq. (8) for rough beds: (a) $k_{s} / k=0.05$ and $i=-0.05$; (b) $k_{s} / k=0.25$ and $i=-0.05$; and (c) $k_{s} / k=0.15$ and $i=-0.10$; and for smooth beds: (d) $i=-0.10$; (e) $i=-0.15$; and (f) $i=-0.17$.

Figure 5 Energy dissipation for sloping rough beds $(i>0)$ : comparison of experimental data with Eq. (8) for: (a) $k_{s} / k=0.05$ and $i=0.05$; and (b) $k_{s} / k=0.35$ and $i=0.15$.

Figure 6 Bed slope effect on the energy dissipation on smooth beds.

Figure 7 Comparison between measured and computed (with Eq. 8) values of the variable $\Delta E_{r}$ for: (a) horizontal bed $(i=0)$; (b) smooth adverse-sloped bed $(i<0)$; and (c) adverse-sloped and sloping rough beds $(i<0$ and $i>0)$. 